\section{THU0307 QT INTERVAL AND ITS CORRELATIONS WITH TRADITIONAL RISK FACTORS OF DEVELOPMENT OF CARDIOVASCULAR DISEASES IN PATIENTS WITH ACTIVE EARLY PSORIATIC ARTHRITIS}

E. Markelova, D. Novikova, T. Korotaeva, I. Kirillova, E. Loginova. V.A. Nasonova Research Institute Of Rheumatology, Moscow, Russian Federation

Background: Cardiovascular diseases (CVD) are leading cause of morbidity and mortality in patients (pts) with psoriatic arthritis (PsA). An abnormally prolonged and shorted QT interval are associate with an increased risk of ventricular arrhythmias and sudden cardiac death.

Objectives: to evaluate QT interval during Holter monitoring and cardiovascular (CV) risk assessment using SCORE (Systematic COronary Risk Evaluation) in early PsA (EPsA) pts.

Methods: We included data of 48 (F.-23) DMARD-naive EPsA pts (according to the CASPAR criteria) with no history of CVD: mean age - 36[ ${ }^{28 ;}$ years, EPsA duration $-6,9{ }^{4 ; 12}$ months, DAS - 3.97 [3.27; 4.1], C-reactive protein - 19.4 [8.8; 37.6]mg/l. Controls subjects were matched by age, sex $(n=48)$. All pts were assessed for traditional risk factors of CVD, ESC guidelines, 201624 hour (24 hour) ECG monitoring were analysed for QT interval corrected for heart rate (QTc). Prolonged QTc was defined as $\geq 460 \mathrm{~ms}$ in women and $\geq 450 \mathrm{~ms}$ in men, short QTc $-<330$ ms. Ten-year risk of CV death was estimated using SCORE algorithms, ESC guidelines, 2016 categorised as low $(<1 \%)$, intermediate $(1 \%$ to $<5 \%$ ), high ( $\geq 5 \%$ to $<10 \%$ ) or very high $(\geq 10 \%)$. Intima-media thickness of the carotid artery (c-IMT) was measured using a high-resolution B-mode ultrasound machine.

Results: QTc interval during the 24 hours was significantly prolonged in EPsA pts when compared to the control group (table 1). We didn't find short or prolong QTc interval in EPsA pts and control group.

Abstract THU0307 - Table 1. QTc interval in EPsA pts and control group

\begin{tabular}{lcc}
\hline Parameters & EPsA pts & Controls \\
\hline QTc $(\mathrm{ms})$, day & $397[376 ; 404]$ & $387,5[370,5 ; 396]^{*}$ \\
QTc $(\mathrm{ms})$, night & $396[377 ; 408]$ & $390[367 ; 396,5]^{*}$ \\
QTc $(\mathrm{ms}), 24$ hour & $395[378 ; 406]$ & $387[370 ; 396]^{*}$ \\
\hline
\end{tabular}

Data are present in median values and interquartile range, ${ }^{*} \mathrm{p}<0,05$ (nonparametric paired Mann-Whitney U-test).

$62.5 \%$ of patients with EPsA were classified as being at low risk 10 year risk of CV death using the SCORE algorithm, 6.25\% pts - intermediate risk, 29.2\% pts high risk, $2.1 \%$ pts - very high risk. Increased cIMT was found in 11 (22.9\%), atherosclerotic plaques - in $15(31.3 \%)$. We found significant correlations between age and QTc duration during the 24 hours $(R=0.48)$, as well as in both day $(R=0.46)$ and night periods $(R=0.45)$, for all $p<0.05$. We didn't find correlations between QTc duration and traditional risk factors of CVD, disease activity of EPsA. Significantly correlations were observed between SCORE level and abdominal obesity $(R=0.43, p<0.05)$, BMI $(R=0.41, p<0.0001), c-I M T(R=0.41, p<0.05)$.

Conclusions: QT interval was significantly prolonged in EPsA pts when compared to the control group. The age of pts was associated with increase of the QTc interval. 29.2\% of patients were classified as being at high risk risk 10 year risk of CV death using the SCORE algorithm. The increase level of SCORE associated with a subclinical atherosclerosis. Combination of prolonged QT interval and carotid atherosclerosis confirms presence of high cardiovascular risk in EPsA pts.

Disclosure of Interest: None declared

DOI: 10.1136/annrheumdis-2018-eular.3685

\section{THU0308 CALPROTECTIN AS A MARKER OF DISEASE ACTIVITY IN PATIENTS WITH NEW ONSET PSORIATIC AND RHEUMATOID ARTHRITIS: CORRELATION WITH ULTRASONOGRAPHIC SYNOVITIS}

${ }^{1}$ G. Sakellariou, G. Lombardi ${ }^{2}$, B. Vitolo ${ }^{1}$, M. Gomarasca ${ }^{2}$, M. Faraldi ${ }^{2}$ R. Caporali ${ }^{1}$, G. Banfi ${ }^{2,3}$, C. Montecucco ${ }^{1} .{ }^{1}$ Chair And Division Of Rheumatology, IRCCS Policlinico San Matteo Foundation, Pavia; ${ }^{2}$ Laboratory of Experimental Biochemistry and Molecular Biology, I.R.C.C.S. Istituto Ortopedico Galeazzi; ${ }^{3}$ VitaSalute San Raffaele University, Milano, Italy

Background: Serum Calprotectin has been tested as a marker of disease activity in psoriatic (PsA) and rheumatoid (RA) arthritis. In RA and in PsA on TNF inhibitors in remission calprotectin correlates with power-Doppler (PD) positive ultrasonographic (US) synovitis, while there is no data on untreated patients with newonset PsA.

Objectives: To investigate the correlation and association between calprotectin and US synovitis in patients with new-onset PsA and in a control group of RA. Methods: Consecutive patients with PsA and a group of age and gendermatched patients with RA, referred to an early arthritis clinic (2005-2014) were included. Demographic and clinical features, including a 44 joint count for tenderness and swelling (TJC, SJC) and C-reactive protein (CRP) were recorded. US of wrists (radiocarpal, intracarpal and ulnocarpal) and MCP joints with grey scale (GS) and PD synovitis scored 0-3 at each site, with a total score from the sum of each site, was available at the same time, as well as serum samples to measure calprotectin concentration. Serum levels of calprotectin were compared by Mann Whitney test in PSA and RA. The correlation between calprotectin, TJC, SJC CRP and US PD and GS was evaluated by Spearman's correlation coefficient, while the association of calprotectin concentrations and PD synovitis by regression analysis. Secondary analyses separating poliarticular and oligoarticular (SJC $\leq 4)$ PsA and using different definitions of synovitis (GS >1, PD >1) were performed.

Results: 156 patients (78 PsA and $78 \mathrm{RA}$ ) were included (RA: male $28.2 \%$, mean (sd) age 51.9 (13.3); PsA male 32\%; mean age 51.7 (13.5)). Patients with RA had significantly higher CRP (median, IQR) $(0.6,0.3-2.1$ vs $0.36,0.3-1, \mathrm{p} 0.04)$, SJC (7, 5-12 vs 6, 3-9, p 0.008), GS (6, 4-11 vs 5, 2-7, p 0.01) and PD (2, 0-9 vs 1 , $0-3$, p 0.003 ) scores. Calprotectin ( $\mathrm{ng} / \mathrm{ml}$, median, IQR) did not significantly diffe in PsA (3123, 2063-4669) and RA (2556, 1615-4441), also when separating poliarticular and oligoarticular PsA. In patients with PsA, calprotectin significantly correlated with GS score (rho 0.340, p 0.007), PD score (rho 0.290,p 0.02) and with the presence of PD (categorical variable) (rho 0.263, p 0.04), while in RA there were no statistically significant correlations. When separating poliarticular and oligoarticular PsA, a significant correlation between calprotectin and GS score (rho 0.369, p 0.01) and PD score (rho 0.363, p 0.02) was confirmed in poliarticular but not oligoarticular disease. In both RA and PsA SJC and TJC did not sig nificantly correlate with calprotectin. Calprotectin showed a statistically significan correlation with CRP in both PsA (rho 0.273, p 0.01) and RA (rho 0.27, p 0.01), showing concurrent validity. In regression analysis, calprotectin levels did not associate with the presence of PD in PsA also when using a more stringent cutoff. Similar results were achieved in RA.

Conclusions: In untreated patients with early onset PsA, but not in RA, calprotectin correlates with US PD-positive synovitis, especially in poliarticular disease. Prospective studies are needed to confirm the use of calprotectin as a biomarker in early inflammatory arthritis.

Disclosure of Interest: None declared

DOI: 10.1136/annrheumdis-2018-eular.6347

\section{THU0309 TEN YEARS FOLLOW-UP STUDY OF CLINICAL DISEASE STATUS AND TREATMENT IN PSORIATIC ARTHRITIS PATIENTS FROM AN OUTPATIENT CLINIC IN SOUTHERN NORWAY}

${ }^{1,2}$ G. Haugeberg, S. Tengesdal ${ }^{3}$, I.J.W. Hansen ${ }^{3}$, B. Michelsen ${ }^{3}$, A. Diamantopoulos ${ }^{1}$, A. Kavanaugh ${ }^{4} .{ }^{1}$ Rheumatology, Martina Hansens Hospital, Bærum; ${ }^{2}$ Research Unit, ${ }^{3}$ Rheumatology, Hospital of Southern Norway Trust, Kristiansand, Norway; ${ }^{4}$ Center for Innovative Therapy, UCSD, San Diego, USA

Background: In the new millennium remission has become an obtainable treatment goal for chronic inflammatory joint disorders, shown in particular for rheumatoid arthritis (RA). This has been attributed to new treatment strategies (early intervention and treat-to-target) and new drugs e.g. biologic Disease Modifying Anti-Rheumatic Drugs (bDMARDs). ${ }^{1}$ For psoriatic arthritis (PsA) there is a lack of longitudinal long term clinical data illuminating potential changes that may have occurred over the years.

Objectives: To explore long term changes in clinical disease status and treatment in PsA patients monitored in an ordinary Norwegian outpatient clinic in the period 2008-2017.

Methods: For each year we collected data from last patient visit recorded in the hospital clinical computer system GoTreatIT Rheuma. Included patients had to fulfil the CASPAR criteria and have peripheral arthritis. Standard clinical data collection included demographic data, clinical measures of disease activity (Disease Activity Score with 28 joint counts [DAS28], Clinical Disease Activity Index [CDAI] and Assessors Global Assessments (AGA) on Visual Analogue Scale 0-100 mm (VAS)) laboratory measures of disease activity (Erythrocyte Sedimentation Rate (ESR), C-Reactive Protein (CRP) and Patient-Reported Outcomes Measures (PROMs) of physical function (MHAQ), morning stiffness and VAS scores for joint pain, fatigue and Patient Global Assessment (PGA). Treatment with prednisolone synthetic DMARDs (sDMARDs) or bDMARDs, was recorded.

Results: Over the 10 years mean annual number of PsA patients monitored was 331 , mean age 58.4 years, disease duration 9.6 years, BMI $27.6 \mathrm{~kg} / \mathrm{m} 2$, females $49 \%$ and current smokers $17.6 \%$. A statistically significant decrease for measures of disease activity for the period 2008-2017 was seen (all $p<0.01$ ): ESR 15.8$10.9 \mathrm{~mm} / \mathrm{hr}$, CRP 7.6-4.1 mg/dl, 28 swollen joints 1.5-0.6, 28 tender joints 3.31.6, DAS28 3.32-2.46, CDAI 10.1-6.8 and AGA 14.5-8.6 mm. No statistically significant changes in PROMs was seen. Mean values for the period was: MHAQ 0.46 , joint pain $36.3 \mathrm{~mm}$, fatigue $44.2 \mathrm{~mm}$, PGA $38.8 \mathrm{~mm}$ and morning stiffness 0.95 hour. From 2008 to 2017 the percentage of patients treated with bDMARDs and/or sDMARDs and/or prednisolone increased from $72.6 \%$ to $80.9 \%$. For the 
10 year period the annual proportion of patients did not significantly change neither for treatment with prednisolone (14.9\%), synthetic DMARDs $(53.0 \%$, Methotrexate $38.5 \%)$ or biologics $(29.9 \%)$, this both for TNF $(28.1 \%)$ and non-TNF inhibitors (1.8\%)

Conclusions: Despite obvious limitations using disease activity measures (28 joint count, DAS28 and CDAI) designed for use in RA, our study indicate that disease activity decreased in our PsA outpatients over the 10 year period. This despite no significant change in proportions of patients treated with sDMARDs and bDMARDs. For PROMs no significant changes was seen. With new available outcome measures designed for use in PsA and more treatment options available e.g. secukinumab (IL17 inhibition) and ustekinumab (IL12/23) and tofacitinib (JAK inhibitor) further improvements in clinical outcomes both for disease activity and patient perception can be expected.

\section{REFERENCE:}

[1] Smolen, et al. ARD 2017;76:960-77

Disclosure of Interest: G. Haugeberg Shareholder of: Diagraphit AS, Grant/ research support from: Unrestricted Grant from Pfizer Norway, S. Tengesdal: None declared, I. J. Hansen: None declared, B. Michelsen: None declared, A. Diamantopoulos: None declared, A. Kavanaugh: None declared

DOI: 10.1136/annrheumdis-2018-eular.4792

\section{THU0310 BIOLOGIC DISEASE-MODIFYING ANTIRHEUMATIC DRUGS IN PSORIATIC ARTHRITIS: A REAL-WORLD COHORT OF 439 PATIENTS}

${ }^{1,2}$ J.-G. Letarouilly, J.-H. Salmon ${ }^{3}$, P. Coquerelle ${ }^{4}$, V. Goeb ${ }^{5}$, M.-H. Guyot ${ }^{6}$, E. Houvenagel ${ }^{7}$, F. Maury ${ }^{8}$, L. Marguerie ${ }^{9}$, G. Morel ${ }^{10}$, G. Baudens ${ }^{11}$, E. SolauGervais $^{12}$, N. Ramdane ${ }^{13}$, R.-M. Flipo ${ }^{1,2}$. ${ }^{1}$ Rheumatology, Lille University Hospital; ${ }^{2}$ Lille University, Lille; ${ }^{3}$ Rheumatology, Maison Blanche Hospital, Reims University Hospitals, Reims; ${ }^{4}$ Rheumatology, Hospital of Bethune, Bethune; ${ }^{5}$ Rheumatology, Amiens University Hospital, Amiens; ${ }^{6}$ Internal medicine, Hospital of Roubaix, Roubaix; ${ }^{7}$ Rheumatology, Hospital of Saint Philibert, Lomme; ${ }^{8}$ Rheumatology, Private Practice, Beuvry; ${ }^{9}$ Rheumatology, Institut Calot, Berck; ${ }^{10}$ Rheumatology, Hospital of Valenciennes; ${ }^{11}$ Rheumatology, Private Practice, Valenciennes; ${ }^{12}$ Rheumatology, Poitiers University Hospital, Poitiers; ${ }^{13}$ Clinical Research and Methodology, Lille University Hospital, Lille, France

Background: For more than 15 years, severe psoriatic arthritis (PsA) has been treated only by TNF inhibitors. Two new Biologic Disease-modifying Antirheumatic Drugs (bDMARDs) have recently arrived on the market with different targets: IL12-23 for ustekinumab and IL 17 for secukinumab. Few studies exist with a large number of patients and with required hindsight.

Objectives: The objective was to assess drug survival in an observational cohort of 630 PsA depending on the line of treatment and to analyse the reasons of discontinuation.

Methods: This is a retrospective, multicentric observational study based on the data of the registry RIC Nord de France, from patients suffering from PSA (CASPAR criteria) and treated by bDMARDs from january 2000 to august 2017. Drug survival is defined as the time from initiation to discontinuation (stop/switch) of biologic therapy on the registry. The number of patients who discontinued each treatment and the duration of therapy were recorded. Using Kaplan-Meier survival curves and Cox-regression analyses [hazard ratios $(\mathrm{HR})$ and $95 \%$ confidence intervals (Cls)], time to discontinuation was compared across cohorts undergoing first-, second- or third-line treatment.

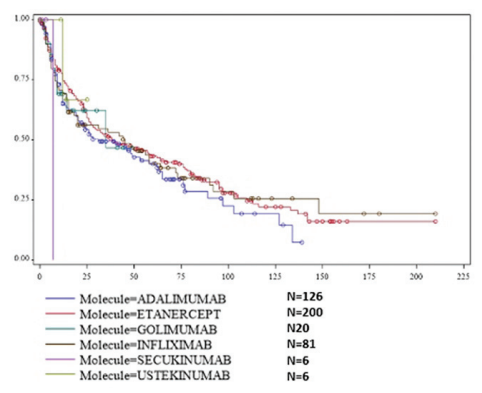

Abstract THU0310 - Figure 1. Drug survival of biotherapies at first-line treatment

Results: Out of 630 PsA, 439 were included with a mean follow up greater than or equal to 6 months. The sex ratio was balanced with $47 \%$ of women. The mean age was 54.5 years old and the body mass index (BMI) was $28.7 \mathrm{~kg} / \mathrm{m}^{2}$. The disease duration was 14.25 years. $51.6 \%$ of patients did not smoke. The DAS-28
CRP was 3.99 at the initiation of the biotherapy. The drug survival of the TNF inhibitors was similar at first-line treatment $(n=439$ patients) (figure 1$)$ and at second-line treatment ( $n=238$ patients). The drug survival of infliximab was statistically longer at third-line treatment $(n=209)(p<0.0001)$, as the drug survival of TNF inhibitors compared to non TNF inhibitor biotherapies (ustekinumab and secukinumab) (p0.011). There was no impact of the age, the sex or the BMI on the drug survival. The discontinuation was mainly due to primary and secondary failure at first-line (respectively $33.33 \%$ and $33.71 \%$ ) and to adverse events at second- and third-line (respectively $30.22 \%$ and $44.55 \%$ ).

Conclusions: The results of the large observational study confirm those of the clinical trials, especially for the patients with failing initial TNF inhibitor therapy.

Disclosure of Interest: None declared

DOI: 10.1136/annrheumdis-2018-eular.4980

\section{THU0311 IMPACT OF SECUKINUMAB TREATMENT ON PSORIATIC ARTHRITIS PATIENTS WITH OR WITHOUT ENTHESITIS AT BASELINE: POOLED DATA FROM TWO PHASE 3 STUDIES (FUTURE 2 AND FUTURE 3)}

${ }^{1}$ J.K. Wallman, G. Schett ${ }^{2}$, I.B. Mclnnes ${ }^{3}$, E. Quebe-Fehling ${ }^{4}$, L. Rasouliyan ${ }^{5}$, L. Pricop ${ }^{6}$, A.E. Fasth ${ }^{7}$, C. Gaillez ${ }^{4}$, on behalf of the FUTURE 2 and FUTURE 3 study groups. ${ }^{1}$ Lund University, Lund, Sweden; ${ }^{2}$ University of ErlangenNuremberg, Erlangen, Germany; ${ }^{3}$ University of Glasgow, Glasgow, UK; ${ }^{4}$ Novartis Pharma AG, Basel, Switzerland; ${ }^{5}$ RTI Health Solutions, Barcelona, Spain; ${ }^{6}$ Novartis Pharmaceuticals Corporation, East Hanover, USA; ${ }^{7}$ Novartis Sverige AB, Täby, Sweden

Background: Enthesitis is a common phenotypic manifestation of psoriatic arthritis (PsA) affecting approximately $70 \%$ of patients (pts) and may be associated with worse outcomes. ${ }^{1}$ Secukinumab (SEC), a fully human monoclonal antibody that selectively neutralises IL-17A, provided significant and sustained improve ment in the signs and symptoms of active PsA, with sustained resolution of enthesitis in Phase 3 studies. ${ }^{2,3}$

Objectives: To report the impact of SEC treatment on efficacy outcome measures in active PsA pts with or without baseline (BL) enthesitis (defined by Leeds Enthesitis Index) using pooled data from the FUTURE 2 (NCT01752634) and FUTURE 3 (NCT01989468) studies over 2 years.

Methods: SEC and placebo (PBO) were administered weekly during the first 4 weeks (wks) followed by subcutaneous maintenance dosing every 4 wks thereafter (PBO until Wk 16/24). The results are reported only for SEC 300 and $150 \mathrm{mg}$ (approved doses). Efficacy outcomes (ACR20/50/70, PASI 90, HAQ-DI, SF-36 PCS and DAS28-CRP) were analysed post-hoc in pts with enthesitis at BL (BLE; $\mathrm{n}=466$ ) or without enthesitis at $\mathrm{BL}$ (No $B L E ; n=246)$. Observed data are presented for binary variables and least-square (LS) means from analysis of covariance for continuous variables.

Results: A total of $65 \%$ of pts had BLE. BL demographics were balanced between the BLE and No BLE groups except for a higher proportion of females and numerically higher tender joint count, disability (HAQ-DI) and lower physical function (SF-36 PCS) in BLE pts than No BLE pts. At Wk 16, improvements in ACR and PASI responses, HAQ-DI, SF-36 PCS and DAS28-CRP were similar in both groups treated with SEC $300 \mathrm{mg}$, but were lower (except for PASI) in BLE pts treated with SEC $150 \mathrm{mg}$ (table 1). Improvements in these outcomes followed a similar trend to Wk 104 in SEC-treated pts (table 1).

Abstract THU0311 - Table 1. Summary of Results with Secukinumab

\begin{tabular}{|c|c|c|c|c|c|c|c|}
\hline & \multirow[t]{2}{*}{ Wk } & \multicolumn{3}{|c|}{ BLE } & \multicolumn{3}{|c|}{ No BLE } \\
\hline & & $300 \mathrm{mg}$ & $150 \mathrm{mg}$ & PBO & $300 \mathrm{mg}$ & $150 \mathrm{mg}$ & PBO \\
\hline \multirow{2}{*}{$\underset{b}{\operatorname{ACR} 20^{a}}$} & 16 & 53.5 & 46.5 & 19.6 & 53.7 & 64.6 & 18.1 \\
\hline & 104 & 56.8 & 52.4 & - & 62.6 & 62.9 & - \\
\hline \multirow{2}{*}{$\underset{b}{\operatorname{ACR} 50^{\mathrm{a}} \text {, }}$} & 16 & 31.3 & 21.4 & 6.7 & 35.8 & 35.4 & 5.6 \\
\hline & 104 & 44.7 & 24.8 & - & 47.3 & 34.3 & - \\
\hline \multirow{2}{*}{$\underset{b}{A C R 70^{a}}$} & 16 & 16.0 & 8.2 & 1.8 & 21.1 & 16.5 & 1.4 \\
\hline & 104 & 26.5 & 15.2 & - & 34.1 & 21.4 & - \\
\hline PASI & 16 & 50.0 & 36.6 & 7.9 & 42.1 & 37.0 & 6.7 \\
\hline $90^{\mathrm{a}, \mathrm{c}}$ & 104 & 67.9 & 59.7 & - & 73.5 & 44.4 & - \\
\hline \multirow[t]{2}{*}{$H A Q-D I^{d}$} & 16 & -0.5 & -0.3 & -0.2 & -0.5 & -0.5 & -0.2 \\
\hline & 104 & -0.5 & -0.4 & - & -0.5 & -0.6 & - \\
\hline SF-36 & 16 & 6.4 & 3.7 & 2.5 & 6.5 & 7.4 & 2.6 \\
\hline $\mathrm{PCS}^{\mathrm{d}}$ & 104 & 7.4 & 4.3 & - & 6.6 & 6.9 & - \\
\hline DAS28- & 16 & -1.5 & -1.05 & -0.5 & -1.35 & -1.6 & -0.5 \\
\hline CRP $^{d}$ & 104 & -1.7 & -1.6 & - & -2.0 & -1.9 & - \\
\hline
\end{tabular}

${ }^{a}$ Response,\%; ${ }^{b}$ At Wk 16/104, $n=144 / 132$ (SEC 300), 159/145 (SEC 150) and 163 (PBO) with enthesitis and $n=95 / 91$ (SEC 300), 79/70 (SEC 150) and 72 (PBO) without enthesitis at BL; ' ${ }^{\mathrm{A}}$ At Wk 16/104, $\mathrm{n}=66 / 56$ (SEC 300), 82/62 (SEC 150 ) and 63 (PBO) with enthesitis and $n=38 / 34$ (SEC 300), 46/36 (SEC 150) and 30 (PBO) without enthesitis at BL (psoriasis subset); d LS mean 